\title{
Editorial
}

\section{Alarms in the operating room}

Historically anaesthetists employed only their senses to monitor patients during the conduct of anaesthesia. Recent developments have made the continuous watching of patient, devices, and every instrumental display virtually impossible. Hearing is an essential communication channel between instrument and user. The human auditory system responds most to the timing of sound stimuli so that a transient increase in firing of auditory neurons may be produced by the termination of sound as well as by its inception. However, instruments are usually equipped with warning noises which need to be interpreted by the user and characteristic sounds which are suitable for the operating room environment have been designed. ${ }^{1,2}$ Loudness can be regulated automatically according to environmental noise ${ }^{3}$ although this seems to be unnecessary in a medical environment. Automated voice messages that can conform to an almost infinite variety of information requirements ${ }^{4}$ are possible but await application to our needs.

Although audible alarms are necessary and have been credited with saving lives some anaesthetists deliberately disarm them. ${ }^{5}$ This implies that they do not believe that audible alarms increase patient safety. Thus the discovery by Loeb et $a l .^{6}$ and Finley and Cohen reported in this journal that some anaesthetists could not identify audible alarms is not surprising. The recognition performance of the subjects would probably be better in their own operating rooms because several communication channels are used simultaneously for tasks in real world situations. The wide disparity between laboratory and real life situations does not justify speculation about the clinical performance of these subjects and the experimental design precludes application to anaesthetists in general. Nevertheless, these investigations draw our attention to an increasingly important aspect of contemporary anaesthesia - communication of urgent information from instrument to anaesthetist.

There are many complaints about traditional warning devices which include the aggravation of false alarms, the wide variety of alarms as well as difficulty in their interpretation. However, research and development continue to encourage the consistent and effective use of audible alarms. For example, "smart" monitoring systems automatically collect and analyze data before communicating $^{7-9}$ and these aid diagnosis and reduce the number of false alarms. In other arrangements the variety has been reduced in a unified system that confines individual warnings to four or five categories, according to physiology, or anaesthesia delivery. In another, the sensitivity of a single warning channel can be reduced. ${ }^{10}$

Encouraging though this is there are some concerns particularly with regard to a reduction in sensitivity to decrease the number of false alarms. A shift to a more conservative criterion is likely to reduce the number of serious situations that will be detected. Smart alarms are an attractive way to improve both the quality of the anaesthetist's working environment and his performance during periods of decreased vigilance and we await reports of clinical testing of such systems. Industry has responded to the needs of the users and there is an urgent need to evaluate these "smart" systems in anaesthesia equipment. Study design methods already exist. " In the meantime, a consortium funded by the Commission of the European Communities has established the Advanced Information in Medicine Programme (AIM). This collaboration of experts, including clinicians, is developing information management and decision support for high patient dependency environments such as operating rooms.

Ergonomics is not a newcomer to clinical anaesthesia journals. ${ }^{12-14}$ The evolution of technology to assist human sensing of patients' needs demands that we take an academic interest in our interaction with the instruments we use. Such considerations are essential for the neophyte and expert, for old and young. Not only will knowledge help anaesthetists to purchase carefully and critically from an industry that is increasingly responsive to their needs but it will ensure that the present displays, controls, and patients are arranged in the best interests of patient safety. 


\section{Avertisseurs en salle d'opération}

Il fut un temps ou l'anesthésiste ne se fiait qu'à ses sens pour surveiller son patient. Tenter de nos jours d'apporter la même attention au patient, aux multiples moniteurs et aux innombrables cadrans et voyants de l'environnement anesthésique tient du prodige. L'ouïe demeure toutefois un canal de communication privilégié entre la machine et son maître. Le système auditif réagit de façon dynamique à la stimulation de telle sorte que ses neurones s'activent aussi bien lorsque qu'un son commence que lorsqu'il cesse. Ainsi, les instruments que nous utilisons émettent des avertissements sonores à être décodés par l'usager ; les caractéristiques des sons ainsi produits peuvent être adaptées à la salle d'opération. ${ }^{1,2}$ L'intensité sonore peut d'ailleurs s'ajuster automatiquement au niveau de bruit environnant ${ }^{3}$ quoique ce soit probablement superflu en salle d'opération. L'utilisation en anesthésie de messages transmis par voix artificielle reste à venir. ${ }^{4}$

Même si on croit que les avertisseurs sonores sont nécessaires et que quelques patients leur doivent la vie, il reste que l'on doit constater que certains anesthésistes (à l'instar d'autres travailleurs ${ }^{5}$ ) les mettent hors-fonction. Doutent-ils de la capacité qu'auraient les avertisseurs sonores d'améliorer la sûreté des patients? Il n'est donc pas surprenant d'apprendre à la lecture de l'article de Loeb et coll. ${ }^{6}$ et de celui de Finley et Cohen plus loin dans ce numéro, que certains anesthésistes se sont avérés incapables de reconnaître des avertissements sonores en laboratoire. Leur performance aurait peut-être été meilleure en salle d'opération où ils utilisent simultanément plusieurs canaux de communication en situation réelle; ainsi ne doit on pas présumer de leur capacité de réaction en clinique et extrapoler à partir d'un test forcément limitatif non plus que de généraliser ses résultats à tous les anesthésistes. Toutefois, il faut saisir l'occasion pour souligner un aspect de plus en plus important de l'anesthessie moderne soit la communication efficace d'informations pertinentes des machines à l'anesthésiste.

Les nombreuses critiques versées au compte des avertisseurs comprennent leur omniprésence, l'irritation causée par le nombre de fausses alarmes et la difficulté d'interprétation de leurs manifestations. Toutefois, l'amé lioration constante des systèmes d'alerte sonore les rendent de plus en plus simples et efficaces. Ainsi les systèmes de monitorage «intelligents " colligent automatiquement et analysent l'information avant d'avertir. ${ }^{7-9}$ Ils facilitent le diagnostic et engendrent moins de fausses alarmes. Certains autres systèmes ont catégorisés les alertes sous quatre ou cinq chapitres selon qu'elles soient de nature physiologique ou aient trait au système anesthésique. Avec d'autres, on peut diminuer la sensibilité d'un seul avertisseur. ${ }^{10}$

Il y a donc moyen de rendre les avertisseurs moins ennuyeux mais la diminution de leur sensibilité afin de réduire le nombre de fausses alarmes risque d'augmenter le nombre d'épisodes importants passant inaperçus. Par contre, les systèmes de monitorage "intelligents" risquent d'améliorer la qualité des conditions de travail de l'anesthésiste et sa performance pendant les périodes de moindre vigilance; c'est ce qu'il faudra vérifier en pratique. A cet effet, il importe d'évaluer adéquatement et au plus tôt tous ces nouveaux systèmes «intelligents " dont l'industrie affuble les appareils d'anesthésie. Notons qu'une commission de la communauté européenne par le biais d'un consortium vient d'établir le Programme avancé d'information médicale. On y retrouve des experts, dont des cliniciens, qui développent un système de traitement de l'information et d'aide au diagnostic approprié aux environnements lourds telle la salle d'opération.

Les journaux d'anesthésie clinique ont déjà traité d'ergonomie. ${ }^{12-14}$ L'évolution technologique dont le but est de nous aider à être plus sensibles aux besoins de nos patients nous oblige à réapprendre pratiquement comment interagir avec toute la machinerie qui nous entoure. Nous devons tous nous y mettre afin de devenir de meilleurs acheteurs parmi la panoplie qui s'offre à nous et afin de $s$ 'assurer que tous ces boutons, pitons et cadrans contribuent en bout de ligne à la sûreté de nos patients.

J.W.R. McIntyre MD

Edmonton, Alberta

\section{References}

1 Patterson RD, Milroy $R$. Auditory warnings on civil aircraft: the learning and retention of warnings. Final contract report 7D/S/0142, MCF Applied Psychology Unit Cambridge, 1980.

2 Stanford LM, McIntyre JWR, Hogan JT. Audible alarm signals for anaesthesia monitoring equipment. Int $\mathrm{J}$ Clin Monit Comput 1985; 1: 51-6.

3 Schiller $E$. Automatic loudspeaker control (ALC): a summary of development and design. 3 Naval Ocean Systems Center, San Diego CA. NTIS Report No: NSC/ TR-434. January 1, 1989.

4 Mclntyre JWK, Nelson TM. Application of automated human voice delivery to waming devices in an intensive care unit. A laboratory study. Int J Clin Monit Comput 1989; 6: 255-62. 
5 McIntyre JWK. Ergonomics: Anaesthetists' use of auditory alarms in the operating room. Int $\mathrm{J}$ Clin Monit Comput 1985; 2: 47-55.

6 Loeb RG, Jones BR, Behrman KH, Leonard RJ. Anesthetists cannot identify audible alarms. Anesthesiology 1990; 73: A539.

7 Pan PH, Van der Aa JJ, Gomez FJ, Van Oostrom JH, Beneken JEW, Gravenstein JS. Smart Anesthesia Monitoring System. Anesthesiology 1990; 73: A450.

8 Orr JA, Westenkow DR. Evaluation of a breathing circuit alarm system based on neural networks. Anesthesiology 1990; 73: A445.

9 Factor M, Sittig DF, Cohn Al, Gelernter D, Miller $P L$, Rosenbaum $S H$. A parallel software architecture for building intelligent medical monitors. Int J Clin Monit Comput 1990; 7: 117-28.

10 Rung GW, Bennett MA. Clinical evaluation of the monitron TM cardiorespiratory monitor: minimizing false alarms. Anesthesiology 1990; 73: A523.

11 Quaglini S, Stefanelli $M$, Barosi $G$, Berzuini A. A performance evaluation of the expert system. ANAEMIA Comp Biomed Res 1987; 21: 307-23.

12 Alnutt $M F$. Human factors in accidents. Brit $J$ Anaesth 1987; 59: 856-64.

13 Westhorpe RN, Cass NM. Ergonomics and monitoring. Anaesth Int Care 1988; 16: 71-5.

14 Weinger $M B$, England $C E$. Ergonomic and human factors affecting anesthetic vigilance and monitoring performance in the operating room environment. Anesthesiology 1990; 73: 995-1021. 